\title{
Identification of Xanthones from Mangosteen Pericarp that Inhibit the Growth of Ralstonia solanacearum
}

Ping Li,${ }^{\dagger}$ Zhongyan Yang, ${ }^{\dagger}$ Bolin Tang, ${ }^{\dagger}$ Qian Zhang, ${ }^{\dagger}$ Zepeng Chen, ${ }^{\perp}$ Jili Zhang,${ }^{\S}$ Jianyu Wei,${ }^{\S}$ Lirong

$$
\text { Sun, } *,+ \text { and Jian Yan*,† }
$$

${ }^{\dagger}$ Key Laboratory of Agro-Environment in the Tropics, Ministry of Agriculture and Rural Affairs; Guangdong Provincial Key Laboratory of Eco-Circular Agriculture; Guangdong Engineering Research Centre for Modern Eco-Agriculture; College of Natural Resources and Environment, South China Agricultural University, Guangzhou, 510642, People's Republic of China

$¥$ Department of Neurobiology, School of Basic Medical Sciences, Southern Medical University, Guangzhou, 510515, People's Republic of China

${ }^{\S}$ China Tobacco Guangxi Industrial Co. Ltd., Nanning, Guangxi, People’s Republic of China

${ }^{\perp}$ Guangdong Provincial Tobacco Monopoly Administration, Guangzhou, Guangdong, People's Republic of China

Corresponding Author

* Lirong Sun, Phone: +86-20-6278-9020 (L.R.S.). E-mail: slr0807@ fimmu.com (L.R.S.).

* Jian Yan, Phone: +86-20-3834-8099 (J.Y.), E-mail: yanjian78@ scau.edu.cn (J.Y.). 

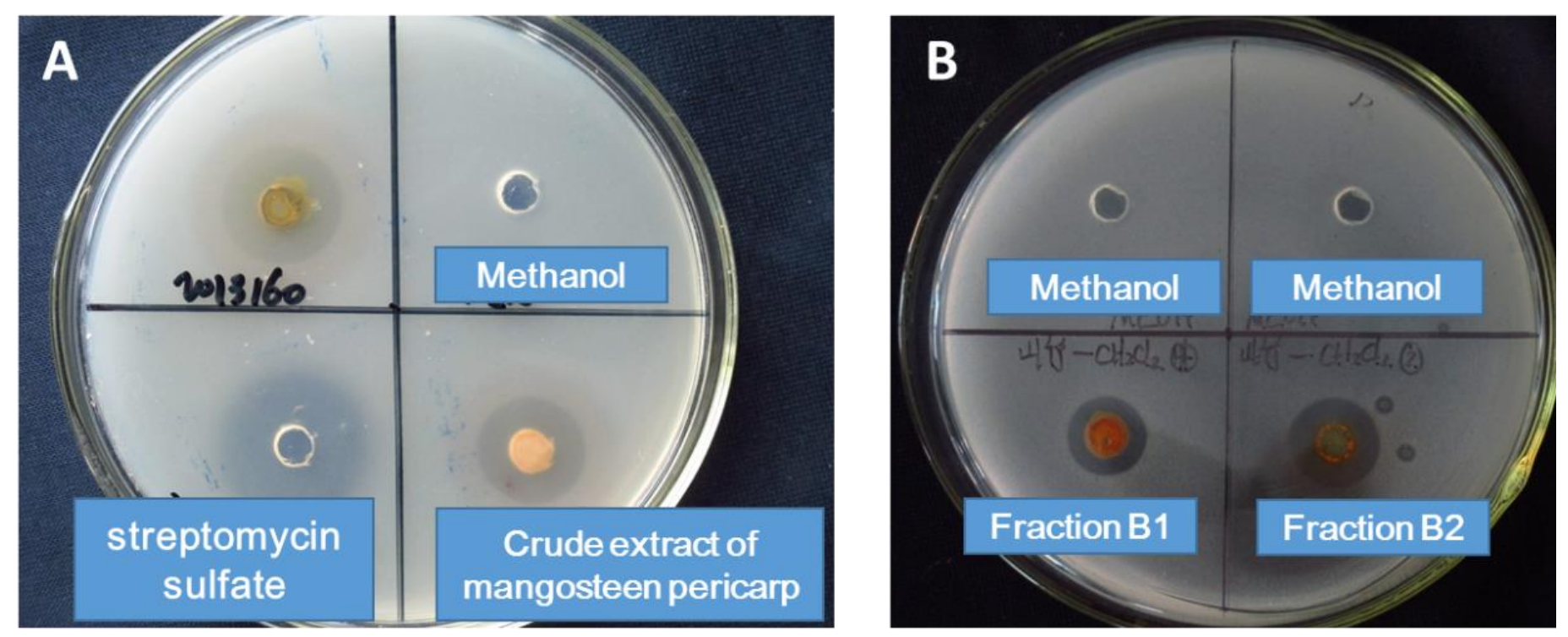

Figure S1. The anti-bacterial activity of crude extract of mangosteen pericarp on agar medium against $R$. solanacearum(A). The anti-bacterial activity of dichlromethane fractions (Fractions B1 and B2) isolated from crude extract of mangosteen pericarp (B). Streptomycin sulfate as positive control and methanol as negative control. 
GM_test_Pos 01

MS2 ES+
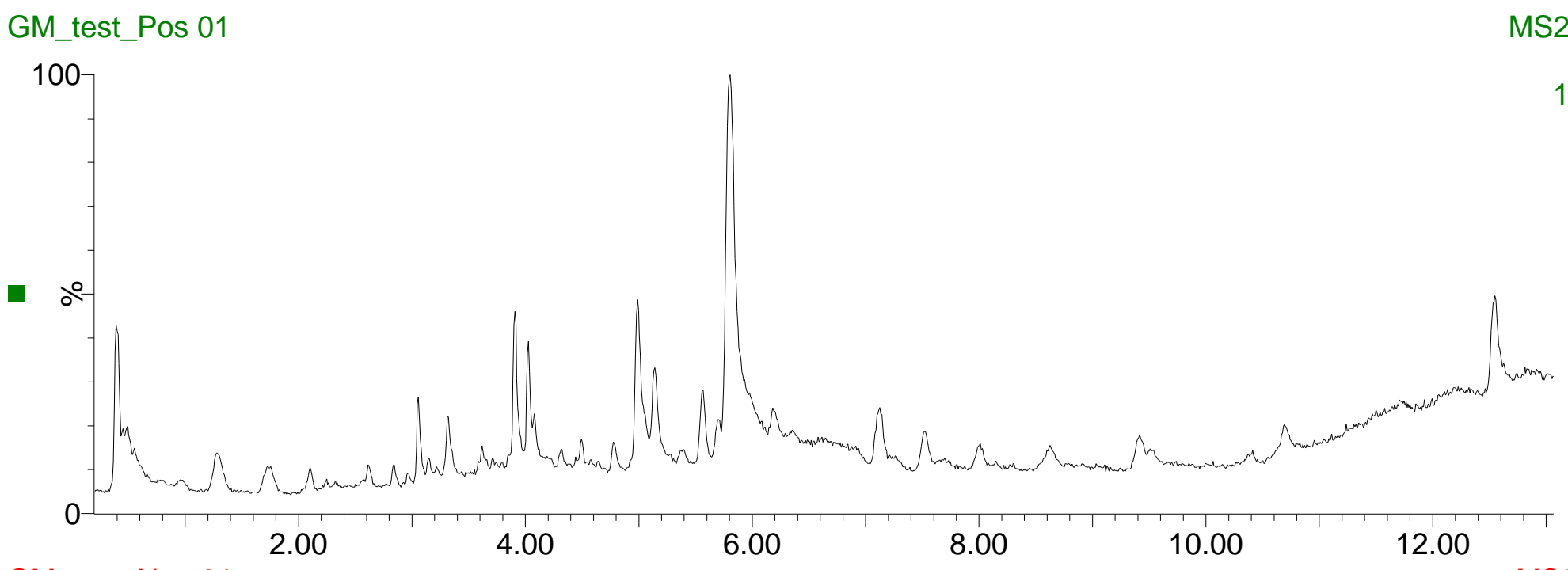

GM_test_Neg 01

8.00

10.00

MS2 ES-

100

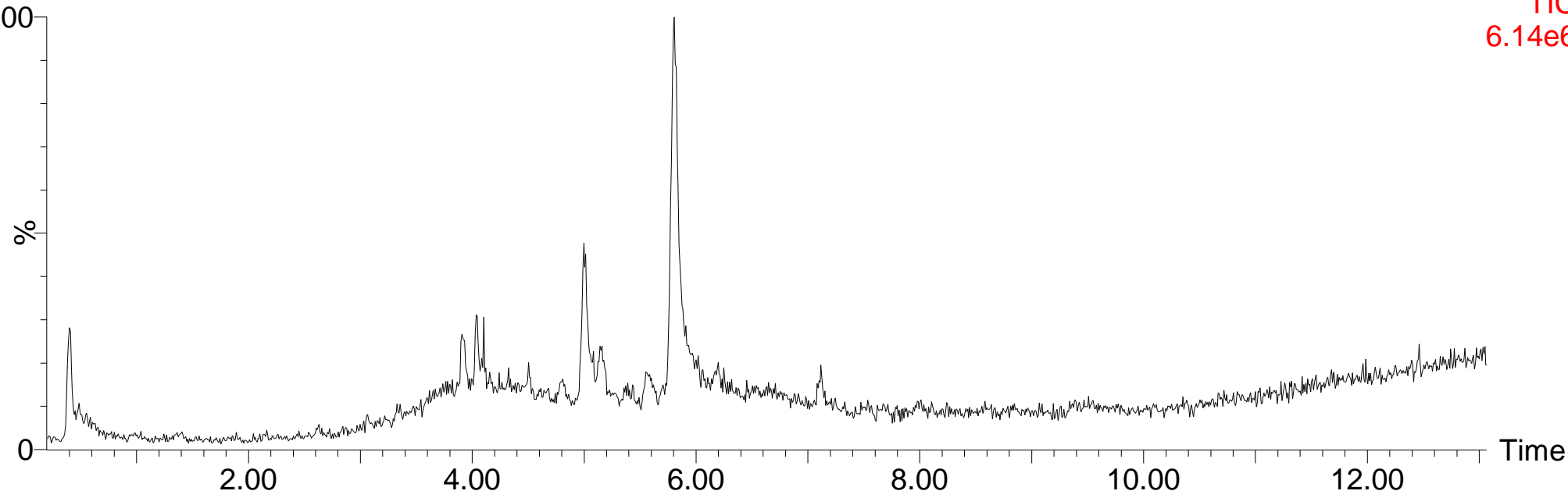

Figure S2. Total ion chromatograph (TIC) of crude extract of mangosteen pericarp at both positive and negative mode. 

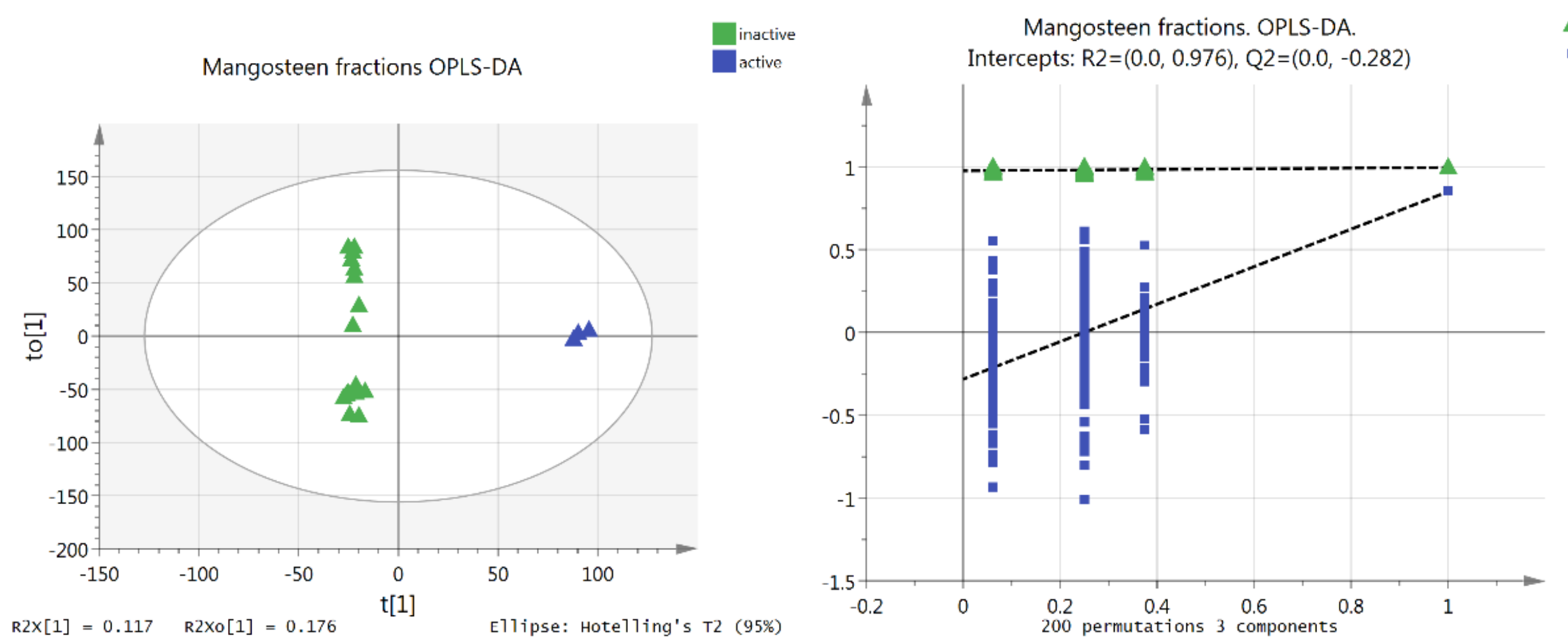

Figure S3. OPLS-DA scores plots of inactive and active fractions (A) and permutation test for the OPLS-DA models (B). 


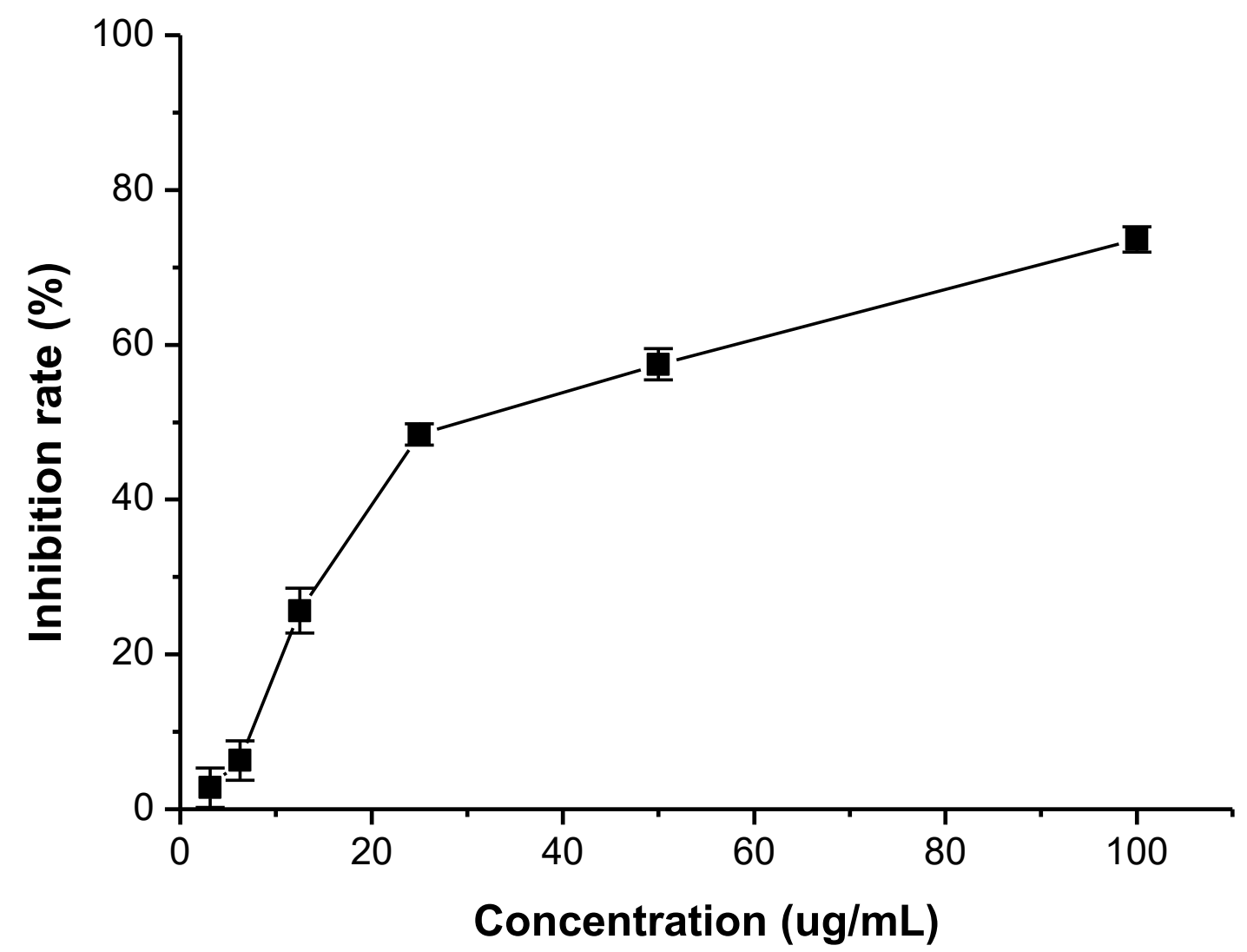

Figure S4. Inhibition Effect of $\gamma$-mangostin against $R$. solanacearum. 


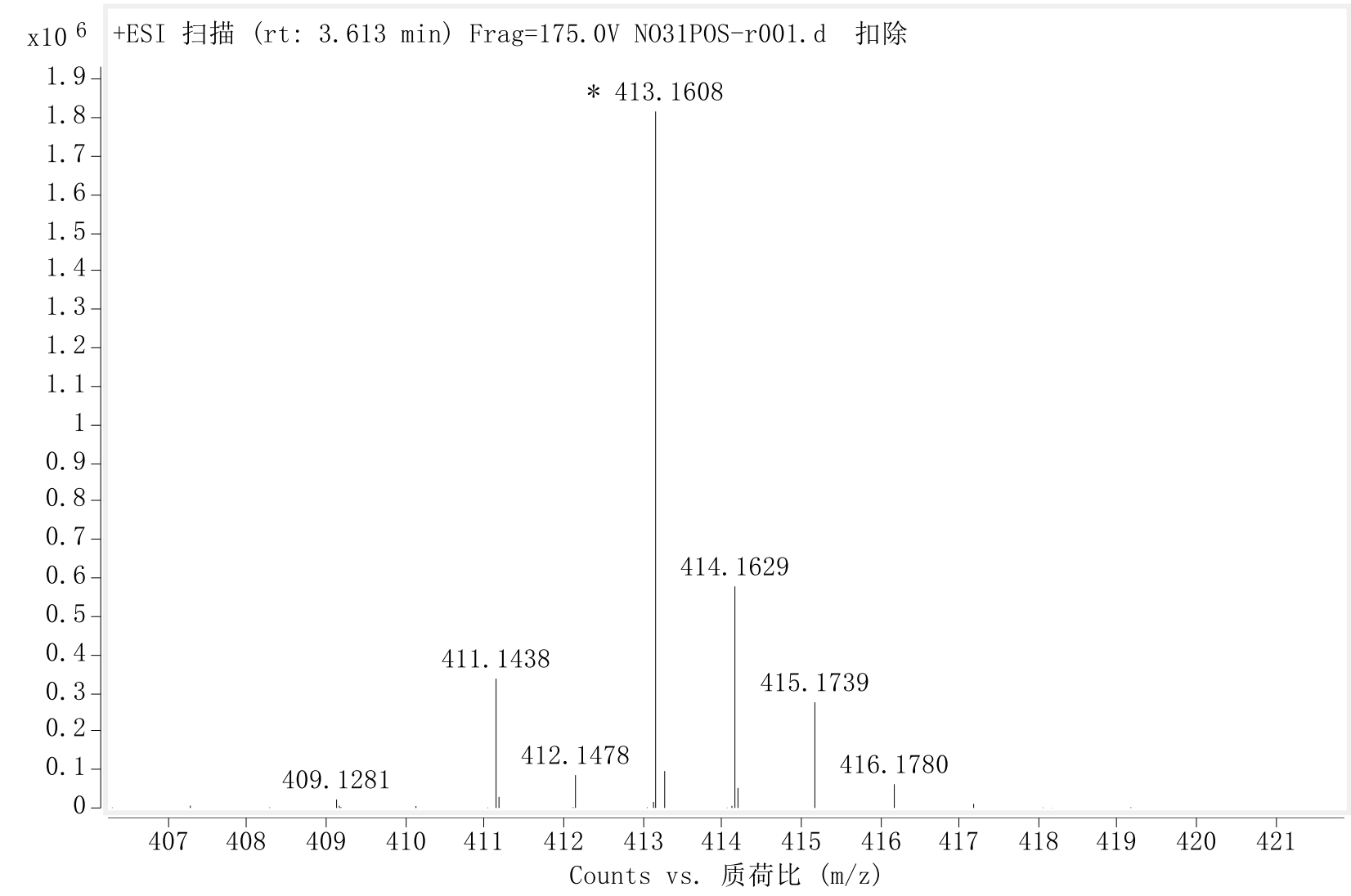

Figure S5. HR-MS of compound 5 


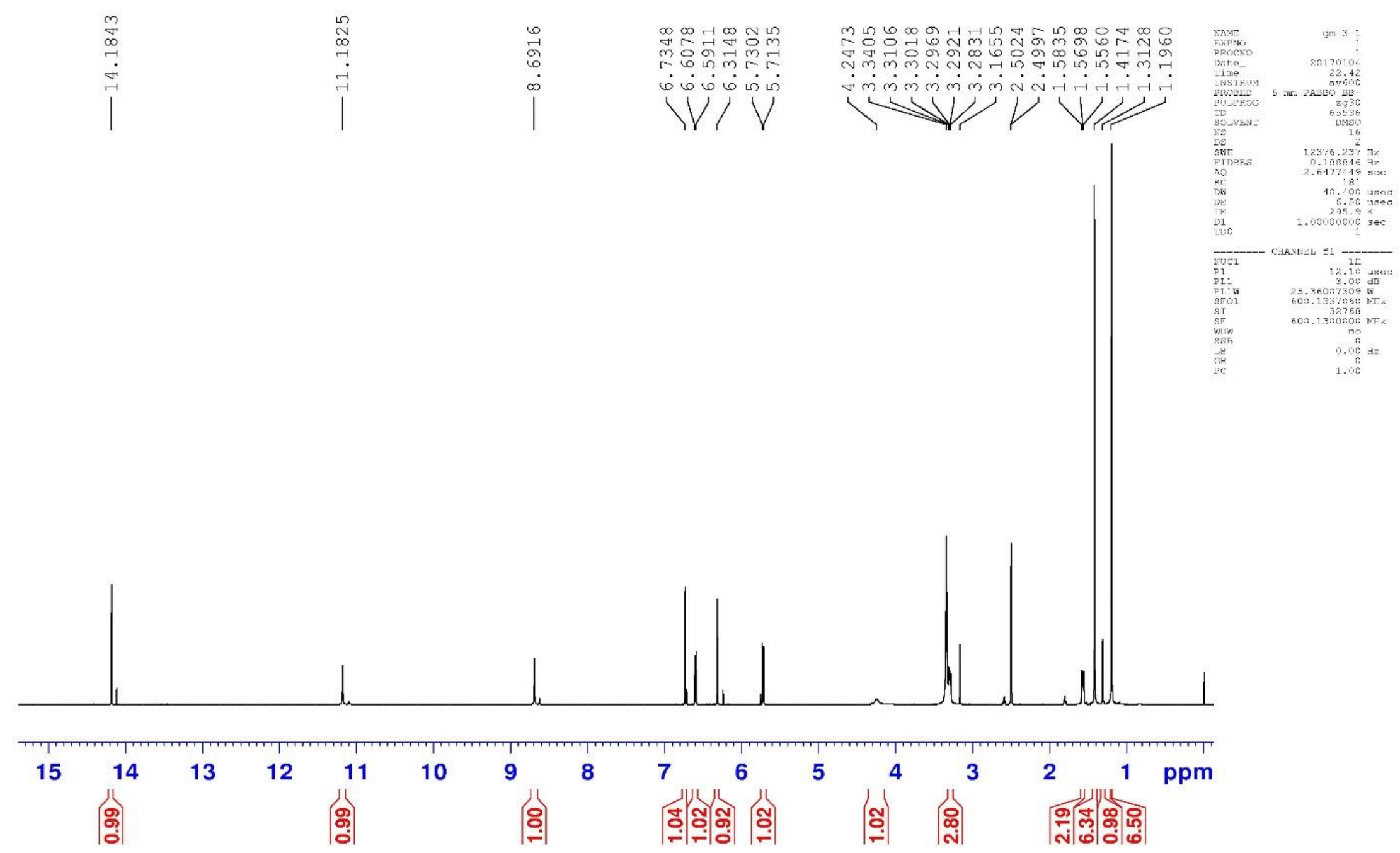

Figure S6. ${ }^{1} \mathrm{H}$ NMR of compound 5. 


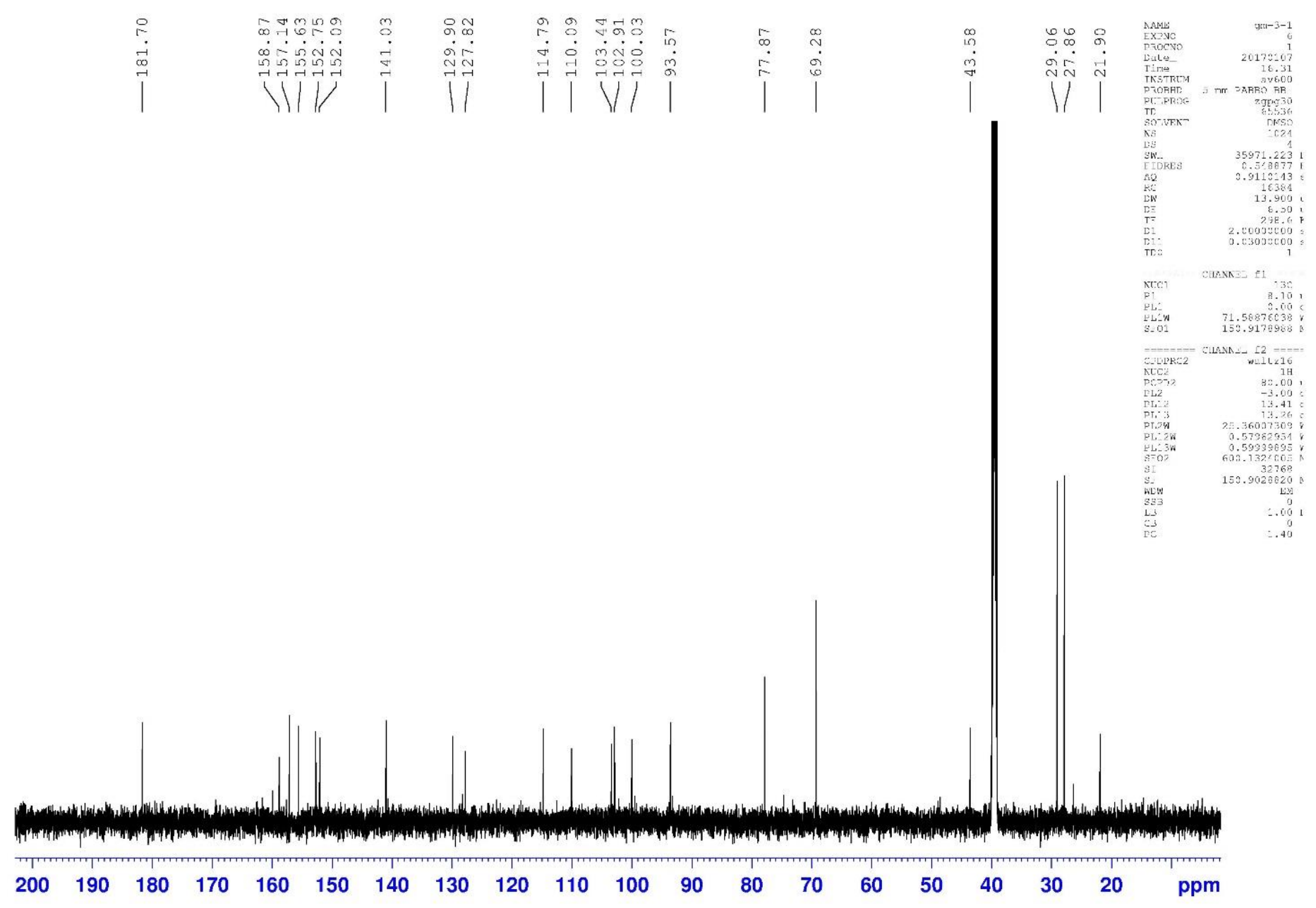

Figure S7. ${ }^{13} \mathrm{C}$ NMR of compound $\mathbf{5}$. 


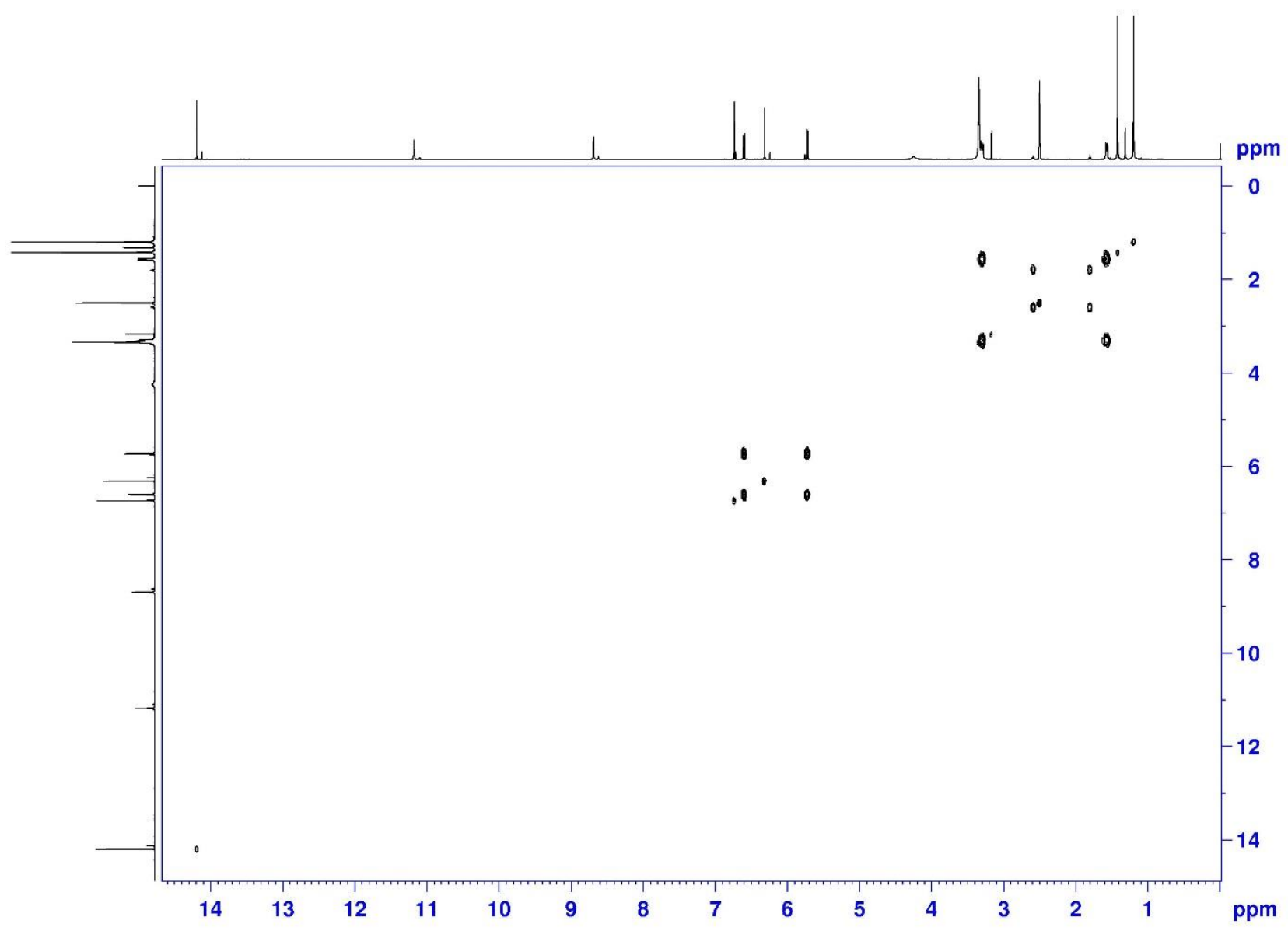

Figure S8. 1H-1H COSY of compound 5. 


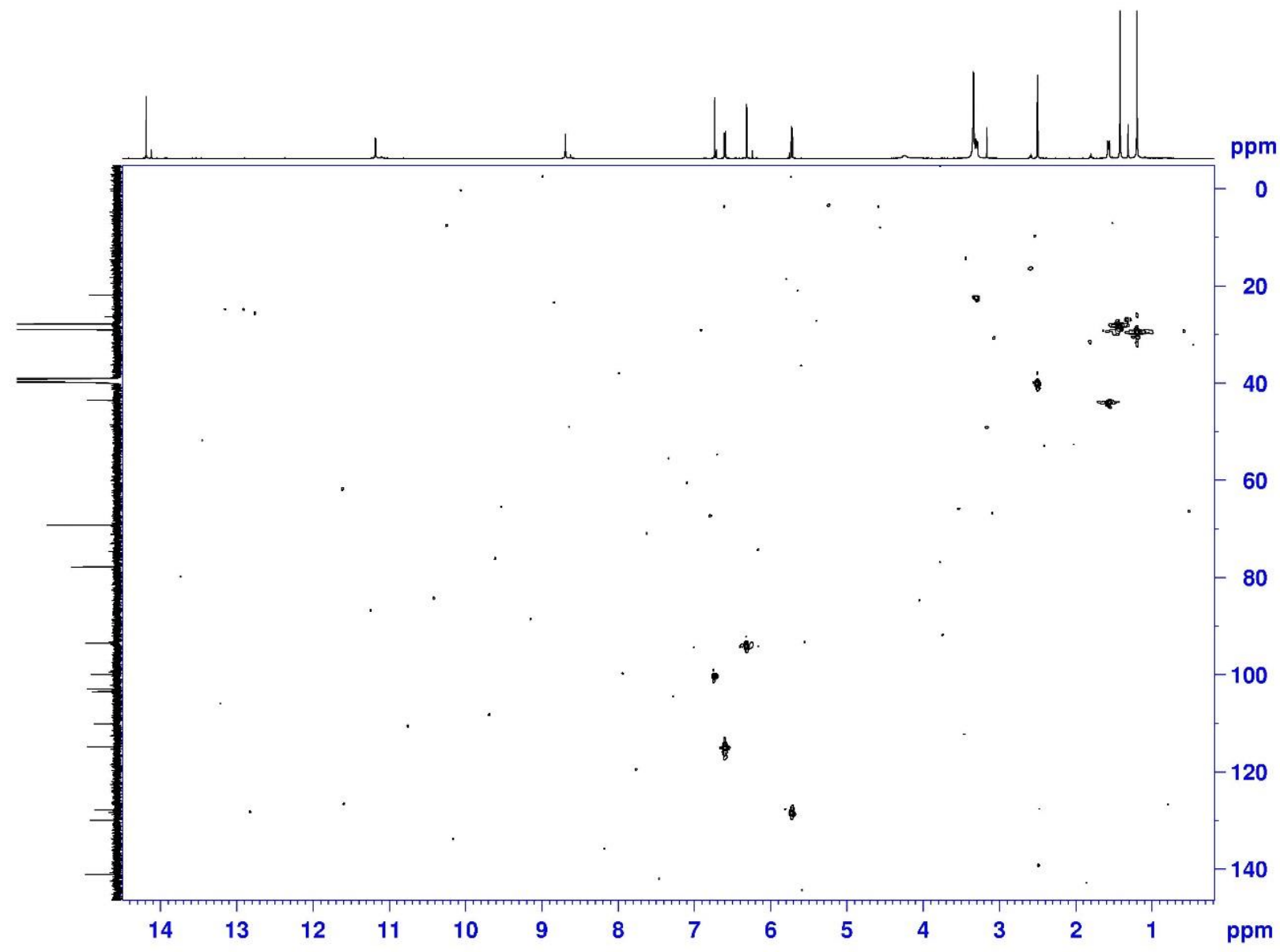

Figure S9. HSQC of compound 5. 


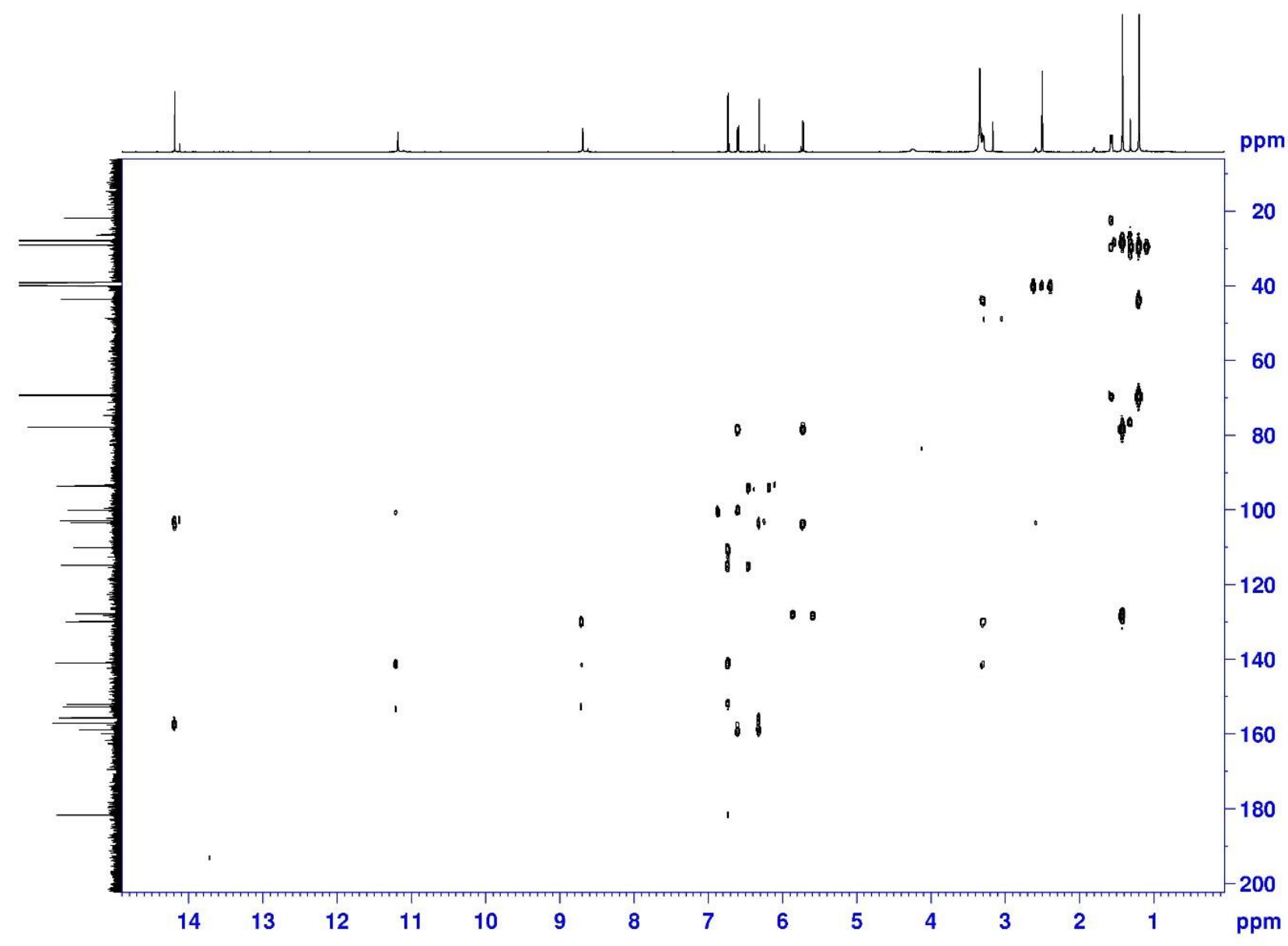

Figure S10. HMBC of compound 5. 


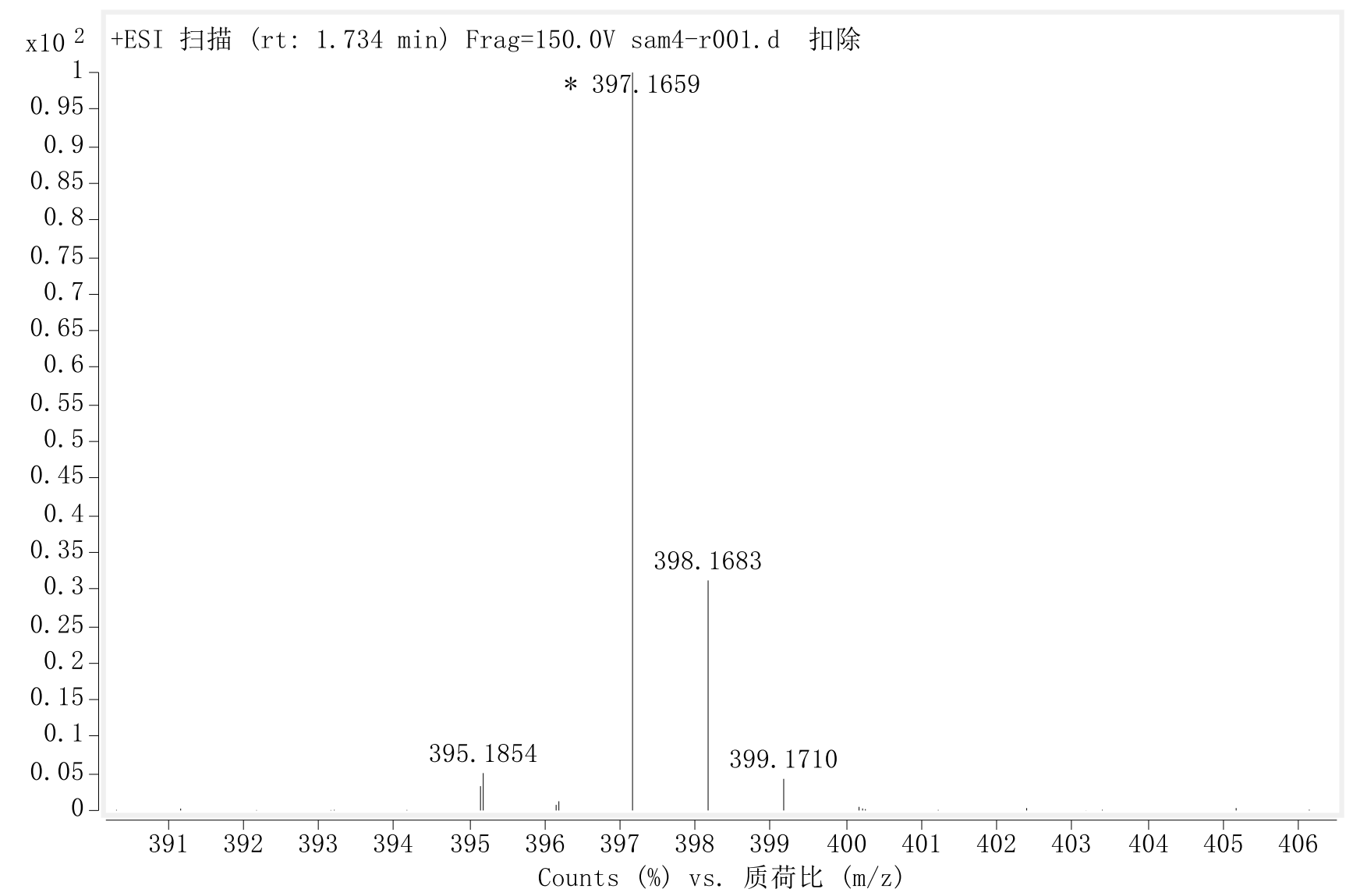

Figure S11. HR-MS of compound 6 


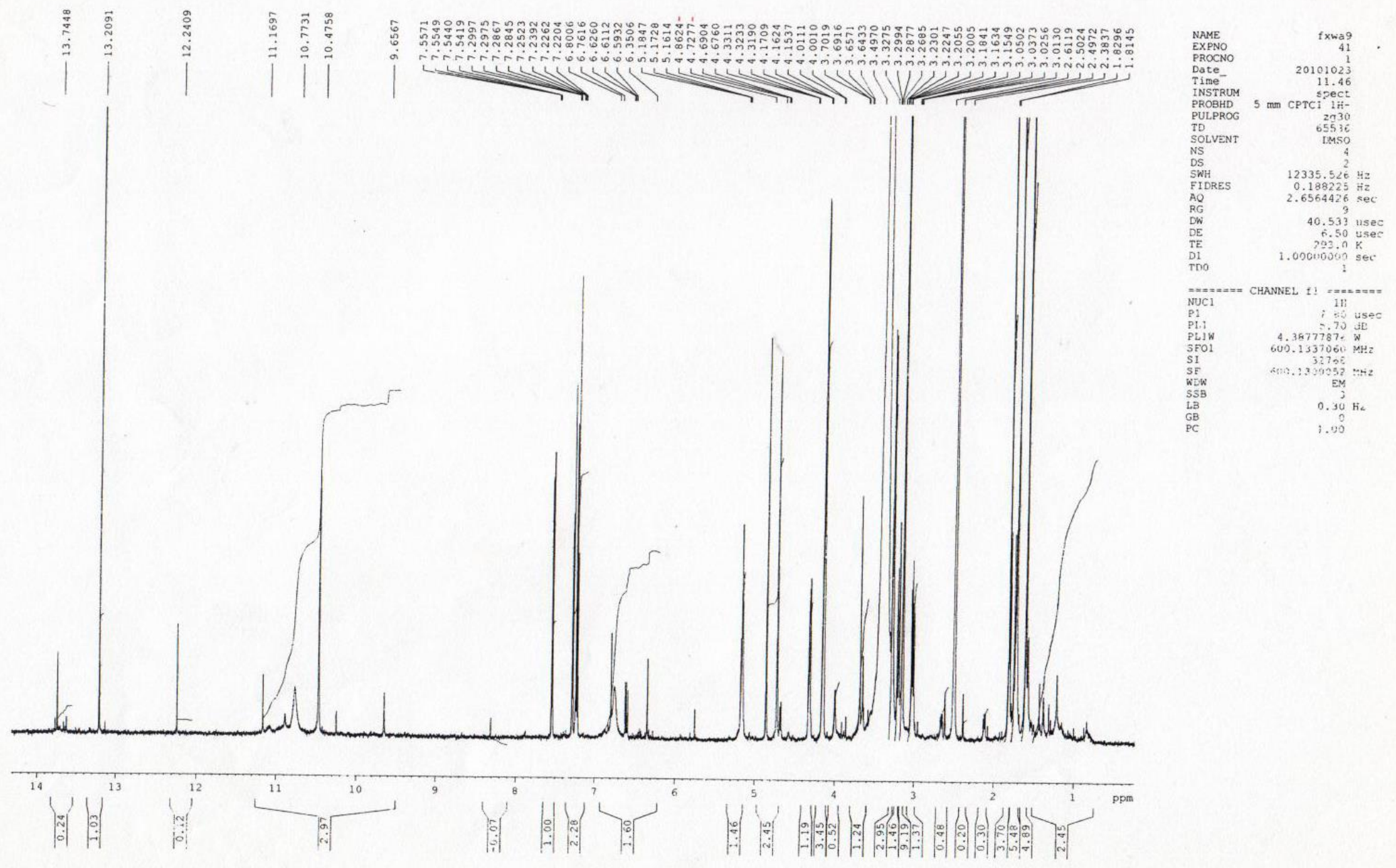

Figure S12. ${ }^{1} \mathrm{H}$ NMR of compound 6. 


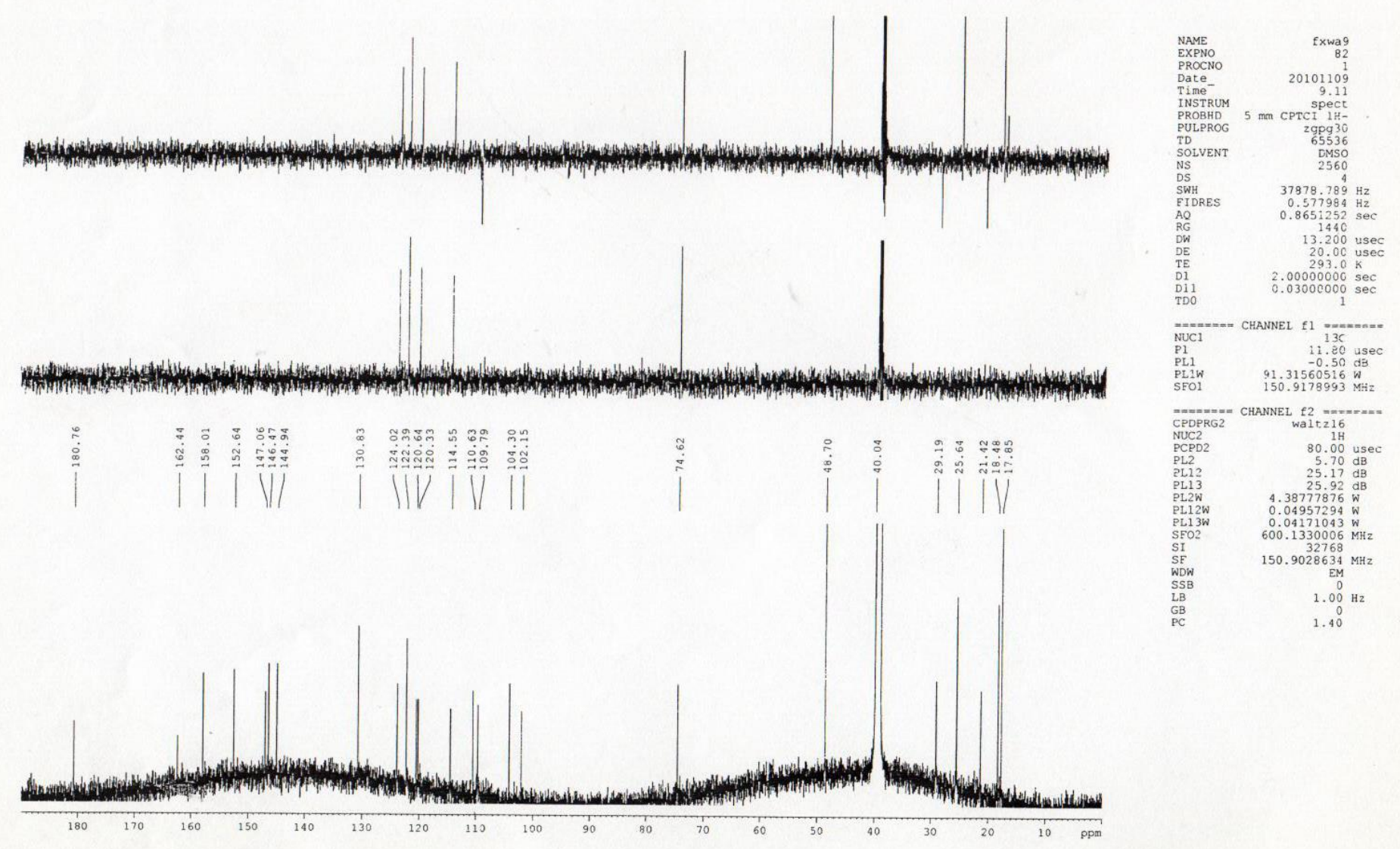

Figure S13. ${ }^{13} \mathrm{C}$ NMR of compound 6 . 


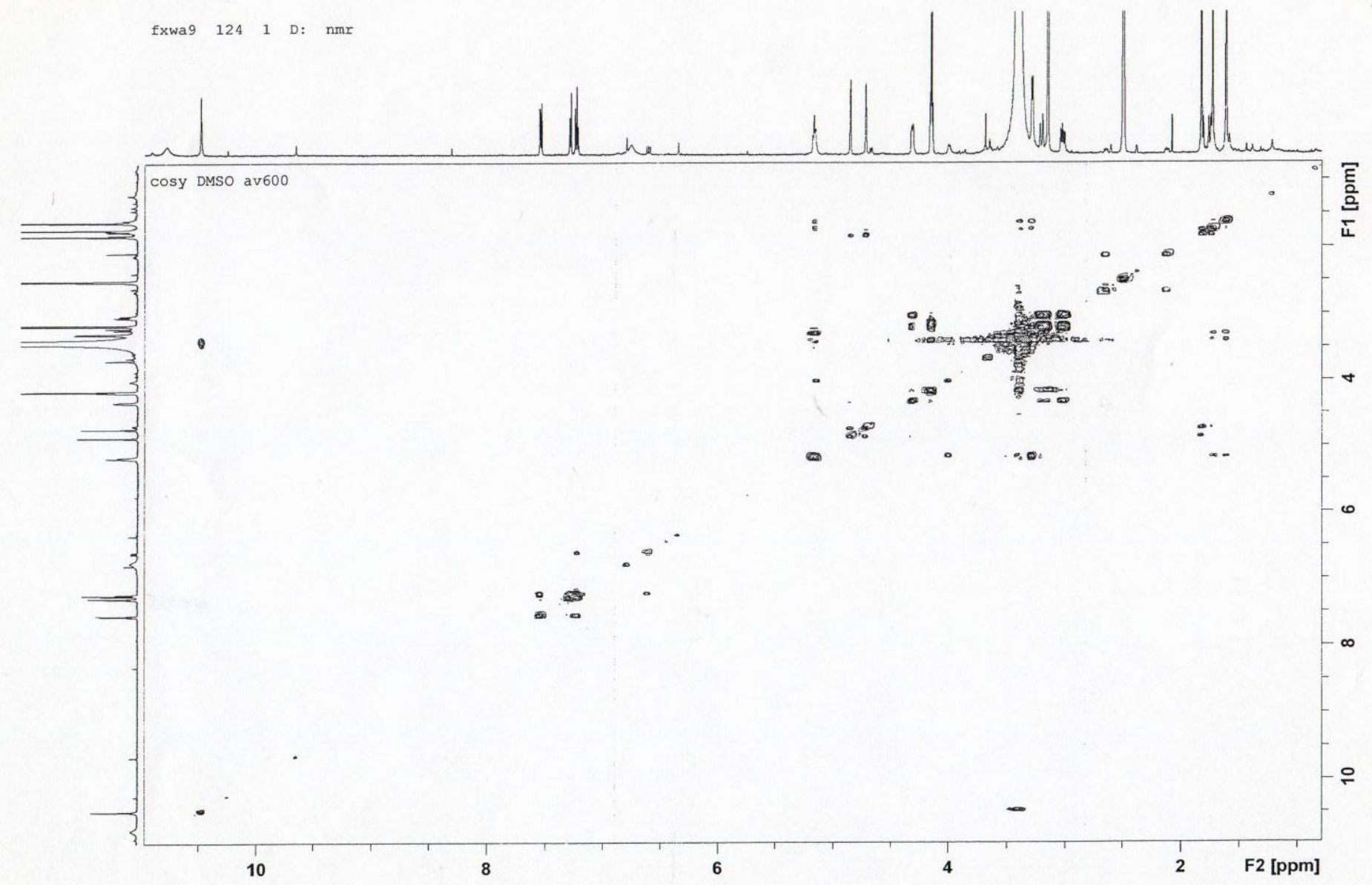

Figure S14. ${ }^{1} \mathrm{H}-{ }^{1} \mathrm{H}$ COSY of compound 6. 


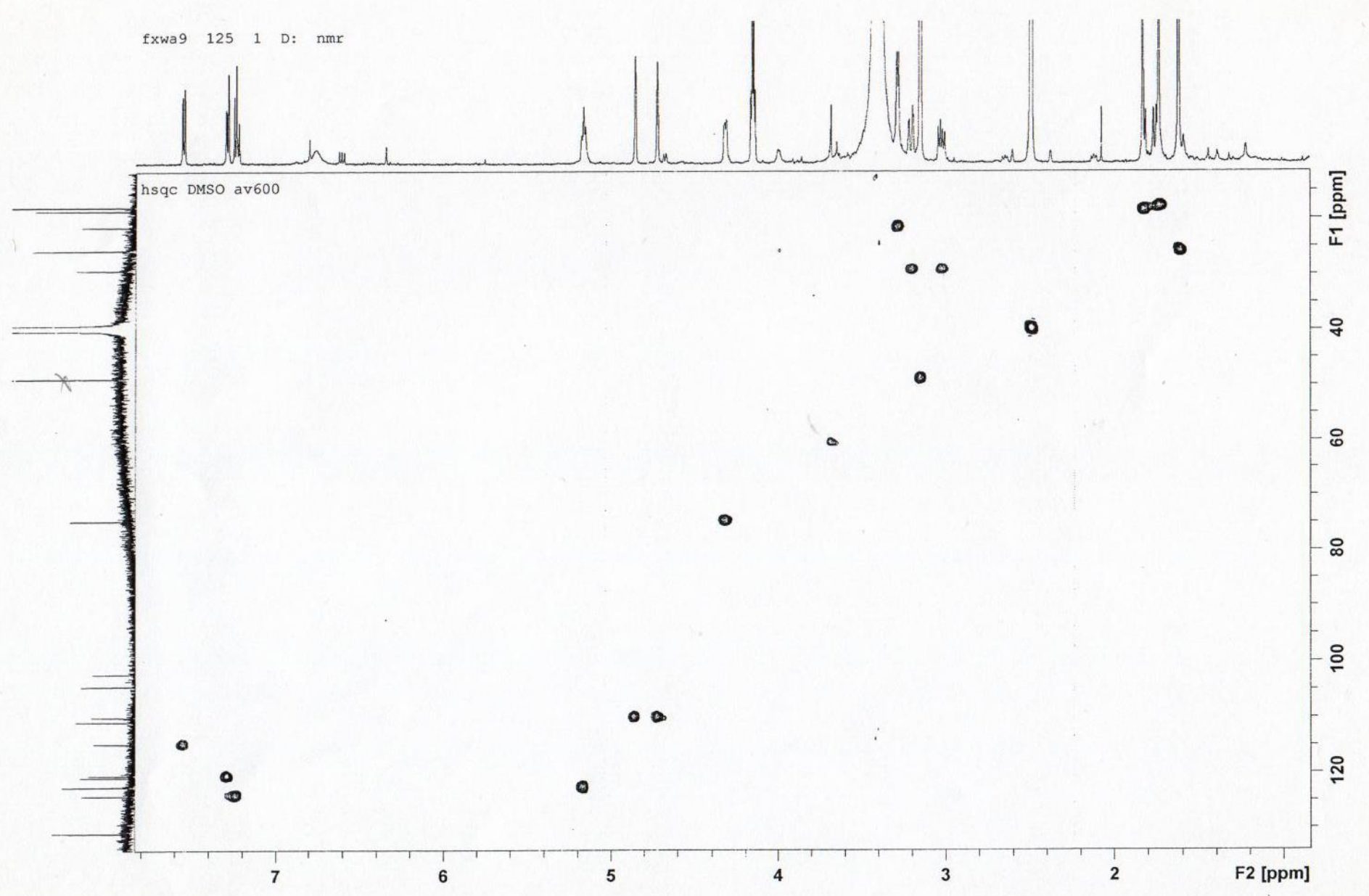

Figure S15. HSQC of compound 6. 


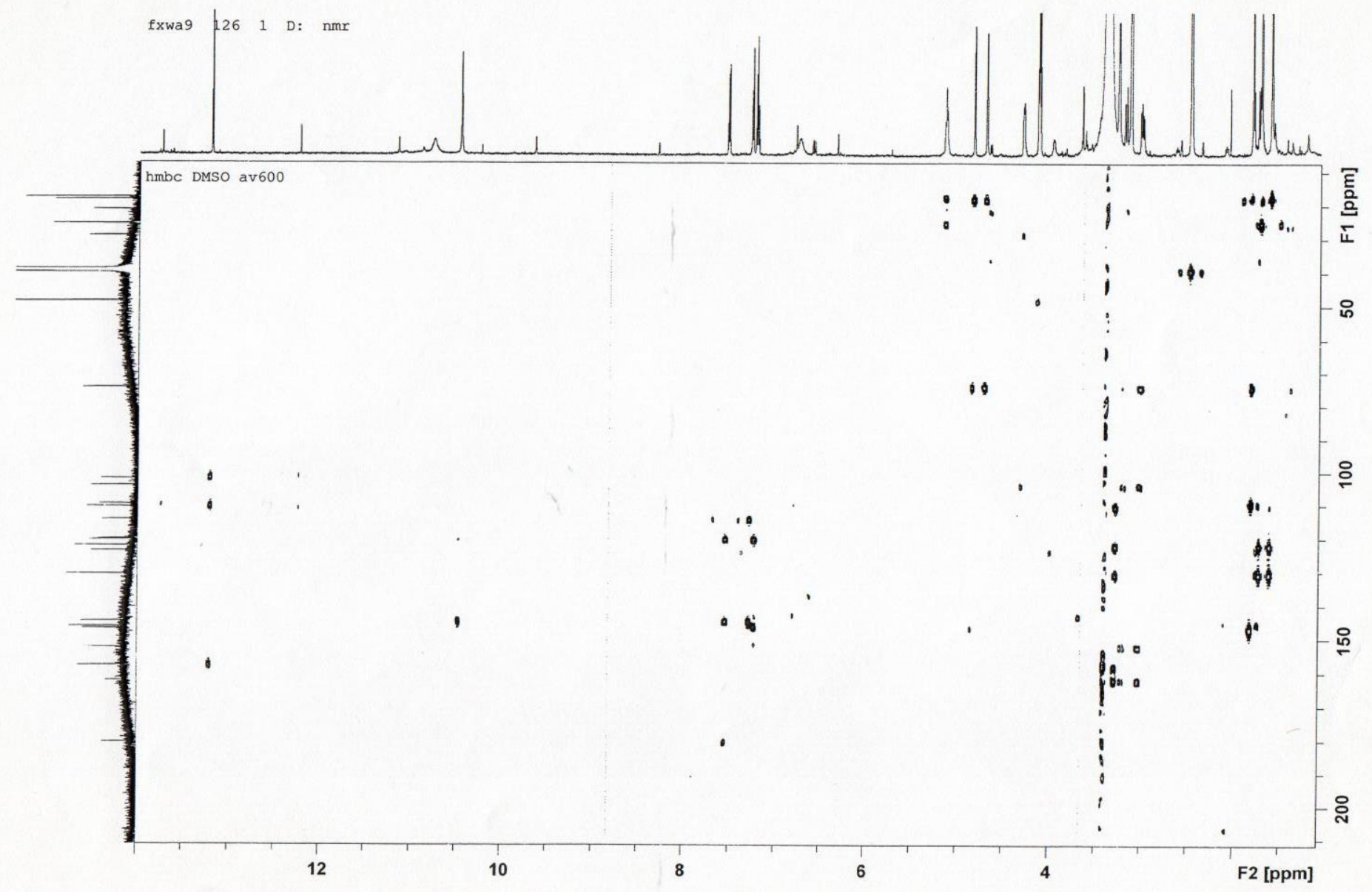

Figure S16. HMBC of compound 6 . 